\title{
Hybridization of TRIZ and CAD-analysis at the conceptual design stage
}

\begin{abstract}
The Theory of Inventive Problem Solving (TRIZ) is a well-known effective methodology to solve the complex problem solving. This paper presents a hybridized TRIZ with CAD-Design analysis through an effective modelling. This modelling helps in solving critical problems for satellite loader parts at the conceptual design stage. The process is applied for critical components in the motorized adjustable vertical platform and proposes a systematic and creative method in the conceptual design process. CAD models for a scissor lifting of motorised satellite platform has been design in the CATIA software and analysed using the ABAQUS to analyse the structure and find the problems of the design, and next optimized using TRIZ inventive tools. The implication of this case study enables optimization of the part in an inventive and effective way.
\end{abstract}

Keyword: Conceptual design; Problem solving; TRIZ; CAD; Analysis 